\title{
Reduced fecal short-chain fatty acids levels and the relationship with gut microbiota in IgA nephropathy
}

Lingxiong Chai ${ }^{1,2}$, Qun Luo ${ }^{1,2}$, Kedan Cai $^{1,2}$, Kaiyue Wang ${ }^{1,2}$ and Binbin $\mathrm{Xu}^{1,2^{*}}$

\begin{abstract}
Background: IgA nephropathy(IgAN)) is the common pathological type of glomerular diseases. The role of gut microbiota in mediating "gut-IgA nephropathy" has not received sufficient attention in the previous studies. The purpose of this study was to investigate the changes of fecal short-chain fatty acids(SCFAs), a metabolite of the intestinal microbiota, in patients with IgAN and its correlation with intestinal flora and clinical indicators, and to further investigate the role of the gut-renal axis in IgAN.
\end{abstract}

Methods: There were 29 patients with IgAN and 29 normal control subjects recruited from January 2018 to May 2018. The fresh feces were collected. The fecal SCFAs were measured by gas chromatography/mass spectrometry and gut microbiota was analysed by 165 rDNA sequences, followed by estimation of $a$ - and $\beta$-diversity. Correlation analysis was performed using the spearman's correlation test between SCFAs and gut microbiota.

Results: The levels of acetic acid, propionic acid, butyric acid, isobutyric acid and caproic acid in the lgAN patients were significantly reduced compared with control group $(P<0.05)$. Butyric acid $(r=-0.336, P=0.010)$ and isobutyric $\operatorname{acid}(r=-0.298, P=0.022)$ were negatively correlated with urea acid; butyric acid( $r=-0.316, P=0.016)$ was negatively correlated with urea nitrogen; caproic acid $(r=-0.415, P=0.025)$ showed negative correlation with $24-h$ urine protein level.Exemplified by the results of a-diversity and $\beta$-diversity, the intestinal flora of IgAN patients was significantly different from that of the control group. Acetic acid was positively associated with $c_{-}$Clostridia $(r=0.357, P=0.008)$, o_Clostridiales( $r=0.357, P=0.008)$ and g_Eubacterium_coprostanoligenes_group $(r=0.283, P=0.036)$. Butyric acid was positively associated with $g \_$Alistipes $(r=0.278, P=0.040)$. The relative abundance of those were significantly decreased in IgAN group compared to control group.

Conclusions: The levels of fecal SCFAs in the IgAN patients were reduced, and correlated with clinical parameters and gut microbiota, which may be involved in the pathogenesis of IgAN, and this finding may provide a new therapeutic approach.

Keywords: IgA nephropathy, Short-chain fatty acids, Gut microbiota

\footnotetext{
*Correspondence: xbb1972@126.com

'Deparment of Nephrology, Ningbo Hwamei Hospital, University of Chinese Academy of Sciences, No.41, Xibei street, Zhejiang Province 315010 Ningbo, China

${ }^{2}$ Life and Health Industry Research Institute, 315010 Ningbo, Zhejiang

Province, China
}

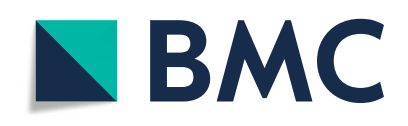

( The Author(s). 2021 Open Access This article is licensed under a Creative Commons Attribution 4.0 International License, which permits use, sharing, adaptation, distribution and reproduction in any medium or format, as long as you give appropriate credit to the original author(s) and the source, provide a link to the Creative Commons licence, and indicate if changes were made. The images or other third party material in this article are included in the article's Creative Commons licence, unless indicated otherwise in a credit line to the material. If material is not included in the article's Creative Commons licence and your intended use is not permitted by statutory regulation or exceeds the permitted use, you will need to obtain permission directly from the copyright holder. To view a copy of this licence, visit http://creativecommons.org/licenses/by/4.0/. The Creative Commons Public Domain Dedication waiver (http://creativecommons.org/publicdomain/zero/1.0/) applies to the data made available in this article, unless otherwise stated in a credit line to the data. 


\section{Introduction}

IgA nephropathy(IgAN) is a clinical and pathological syndrome with heterogenous manifestation and progression [1]. It is the most common primary glomerular diseases in the world and an important cause of end stage renal disease (ESRD) in China [2, 3]. The annual incidence of IgAN is about 2.5 people per 100,000, with the highest incidence in East Asia [4], and more than $50 \%$ of patients may develop ESRD after 25-30 years [5]. However, the pathogenesis of IgAN still has not been completely elucidated currently.

More and more studies have confirmed that there is a close correlation between the kidney and gut microecology, called the "gut-kidney axis" [6, 7]. Recent study has shown that the interaction of microbial, genetic, and dietary factors are thought to induce changes in the function of the intestinal mucosal immune system and promote the progression of IgAN [8].

With the intensive research on gut microecology, the metabolites of gut microbiota are also considered to be important substances involved in the regulation of human vital activities and metabolism. Short-chain fatty acids (SCFAs), including acetic acid, propionic acid, butyric acid and so on, can enter the blood circulation and act as signaling molecules to exert biological effects on peripheral tissues $[9,10]$. Patients with chronic kidney disease have a significantly different gut microbiota with decreased levels of SCFAs $[11,12]$. Previous study has shown that SCFAs exerts its effects on the "entero-renal axis" mainly through G protein-coupled receptors (GPR) and direct inhibition of histone deacetylase (HDAC) [13]. In vivo studies [14, 15], SCFAs, especially acetate and butyrate, were found to inhibit the proliferation of glomerular mesangial cells induced by lipopolysaccharides (LPS) and high glucose in gram-negative bacteria via GPR, and then reversed the production of reactive oxygen species (ROS) and malondialdehyde (MDA) but increased levels of antioxidant enzyme superoxide dismutase (SOD).

There are scarce clinical studies on SCFAs in patients with IgAN. In this study, the changes of fecal SCFAs were measured by gas chromatography mass spectrometry(GC/MS), and their correlations with intestinal flora and clinical indices were investigated to provide new ideas and methods for the role and mechanism of gutrenal axis in IgAN.

\section{Materials and methods Subjects}

Twenty-nine patients with IgA nephropathy, 12 males and 17 females, aged $38.21 \pm 11.80$ years, BMI $23.35 \pm$ $2.88 \mathrm{~kg} / \mathrm{m}^{2}$, who were followed up at Ningbo Hwamei Hospital of the Chinese Academy of Sciences from
January 2018 to May 2018, were enrolled in this study. The tablets they taken during treatment were renin angiotensin inhibitors/angiotensin recetor blockers (28, $96.55 \%)$, calcium channel blockers (5, 17.24\%). A total of 29 age- and sex-matched normal controls were enrolled from Health Check Center, including 12 males and 17 females, aged $38.69 \pm 9.90$ years, and BMI $23.37 \pm 3.46 \mathrm{~kg} / \mathrm{m}^{2}$ (Supplement Table S1). Inclusion criteria of IgAN group: patients aged $\geq 18$ years and diagnosed with IgAN proved by renal biopsy; complete clinical and pathological data; glucocorticoids and/or immunosuppressive agents not taken before renal biopsy. Exclusion criteria: (1) patients diagnosed with secondary IgAN; (2) patients combined with other renal diseases; (3) pregnant or lactating women; (4) patients with infection or stress; (5) patients with acute renal injure or malignant hypertension (diastolic blood pressure $\geq 130$ $\mathrm{mmHg}$ ); (6)patients treated with antibiotics and/or functional food (probiotics and/or prebiotics) in the past three month; (7)patients with type 1 or type 2 diabetes mellitus, neurological or gastro-intestinal diseases; (8) acute myocardial infarction or stroke in the previous six months, severe liver disease, malignancies or with other known immunological or autoimmune disease. All the participants confirmed that there were no remarkable changes in meals and medication for over 1 month. This study was approved by the Ethics Committee of the Ningbo Hwamei Hospital (No.PJ-NBEY-KY-2018-04601 ), and the informed consent form was obtained from each subject.

\section{Clinical data collection}

Fasting blood samples were collected from each subject. Urine samples of $24 \mathrm{~h}$ were collected from IgAN patients. ADVIA 2400 automatic biochemical analyzer (Siemens, Erlangen, Germany) was used to measure serum creatinine, urea nitrogen, uric acid, albumin, total cholesterol, low-density lipoprotein, triglycerides and $24 \mathrm{~h}$ - urine protein. The estimated glomerular filtration rate (eGFR) was calculated by CKD-EPI equation (Levey et al. 2009) [16]. The BC-6800 automatic five-division blood cell analyzer (Mindray, Shenzhen, China) was used to detect white blood cell(WBC) count, neutrophil count, and lymphocyte count, hemoglobin, platelet count, .

\section{Collection of fecal sample}

Fresh fecal samples were collected, quickly placed in ice boxes, and transferred to the laboratory for subpackaging. After sub-packing, the samples were quickly transferred to a $-80{ }^{\circ} \mathrm{C}$ cryogenic refrigerator for freezing, and the collection and packaging process was completed within $30 \mathrm{~min}$. 


\section{Fecal SCFA detected by GC/MS}

Accurately weigh $50 \mathrm{mg}$ of the fecal sample into a 1.5 $\mathrm{ml}$ centrifuge tube, add grinding beads, and then add $300 \mu \mathrm{L}$ of ultrapure water, and homogenize for $2 \mathrm{~min}$. Centrifuge at $18,000 \mathrm{~g}$ for $20 \mathrm{~min}$ at $4{ }^{\circ} \mathrm{C}$. Take $200 \mu \mathrm{L}$ of supernatant and $50 \mu \mathrm{L}$ of concentrated sulfuric acid diluted with $50 \%$ water, then add $200 u$ ul of ether solution (including internal standard $5 \mu \mathrm{g} / \mathrm{ml}$ ), shake for $1 \mathrm{~min}$, then sonicate for $1 \mathrm{~min}, 4^{\circ} \mathrm{C}, 12,000 \mathrm{rpm}$, centrifuge for $20 \mathrm{~min}, 4{ }^{\circ} \mathrm{C}$, stand for $10 \mathrm{~min}$ ether in the upper layer was transferred to an autosampler vial.

Each $1-\mu \mathrm{L}$ of the sample was injected in split mode with a split ratio 5:1 into an Agilent 7890B gas chromatograph coupled with a Pegasus HT time-of-flight mass spectrometer (Leco Corporation, St. Joseph, MI). Separation was achieved on a DB-FFAP column, with helium(99.9999\%) at a constant flow rate of $1 \mathrm{ml} / \mathrm{min}$. All of these processes were conducted in Metabo-Profile Biotechnology Co.,Ltd. in Shanghai, China.

\section{Microbiome/metagenome-wide association studies of gut microbiota}

DNA was sequenced using $16 \mathrm{~S}$ rDNA amplicons. DNA was extracted from fecal samples using the PowerSoil ${ }^{\circ}$ DNA Isolation Kit (MO BIO). $16 \mathrm{~S}$ rDNA/ITS genes of distinct regions (16SV3-V4) were amplified used specific primer with the barcode. All PCR reactions were carried out with Phusion ${ }^{\bullet}$ High-Fidelity PCR Master Mix (New England Biolabs). DNA purity and integrity were analyzed by agarose gel electrophoresis; purified with Gene$\mathrm{JET}^{\mathrm{mm}}$ Gel Extraction Kit (Thermo Scientif).Sequencing libraries were generated using $\mathrm{TruSeq}^{\circ}$ DNA PCR-Free Sample Preparation Kit (Illumina, USA). The library quality was assessed on the Qubit@2.0 Fluorometer (Thermo Scientific). At last, the library was sequenced on an Ion $\mathrm{S}^{\mathrm{Tw}}$ platform. It was conducted in Zhejiang Tianke High Technology Development Co.Ltd.(Zhejiang, China).

\section{Statistical analysis}

SPSS version 22 (Chicago, IL, US) was used for data analysis. The data were showed as the means \pm standard deviations (SD). Student's $t$ test was used for the continuous data. The continuous non-parametric data were presented as medians (interquartile ranges, IQRs), and were compared using Mann-Whitney U test. Microbial analysis was performed to estimate $\alpha$-diversity and $\beta$-diversity and to compare the differences in microbial community structure between IgAN group and control group. To determine the significantly different taxa between two groups, linear discriminant analysis (LDA) was performed using an online utility [17]. Significantly different bacteria with LDA scores $\geq 2.0$ were diagrammed on cladogram. Correlations analysis were performed with spearman's rank test. Two-tailed $\mathrm{p}<$ 0.05 was considered statistically significant.

\section{Results \\ Comparison of clinical parameters between two groups}

The age ,gender and BMI data were matched between two groups. Serum creatinine, urea nitrogen and uric acid were increased in IgAN group $(P<0.05)$. The level of eGFR showed no significance between normal control and IgAN groups. Total Cholesterol, triglyceride and LDL levels as well as leukocytes and neutrophils were significantly higher in the IgAN group compared with normal controls $(P<0.05)$. The level of serum albumin was significantly decreased in $\operatorname{IgAN} \operatorname{group}(P<0.05)$. Lymphocyte, hemoglobin, platelet and, glucose showed no significance between control and IgAN groups (Table 1).

Fecal short-chain fatty acid levels between two groups The SCFAs such as acetic acid, propionic acid, butyric acid, isobutyric acid, valeric acid, isovaleric acid, and caproic acid were quantified by GC/MS (Table 2). The levels of acetic acid, propionic acid, butyric acid, isobutyric acid and caproic acid in the IgAN patients were significantly reduced compared with control $\operatorname{group}(P<0.05)$.

Correlation analysis of fecal SCFAs and clinical parameters As shown in Table 3 , butyric acid $(r=-0.336, P=0.010)$ and isobutyric $\operatorname{acid}(r=-0.298, P=0.022)$ were negatively correlated with urea acid; butyric acid $(r=-0.316$, $P=0.016)$ was negatively correlated with urea nitrogen; caproic $\operatorname{acid}(\mathrm{r}=-0.415, P=0.025)$ showed negative correlation with 24-h urine protein level. Meanwhile, a strong inverse relationship between SCFA and serum lipids. Propionic acid, butyric acid and isobutyric acid showed negative correlation with total cholesterol $(P<0.05)$. Butyric acid, isobutyric acid and valeric acid showed negative correlation with triglyceridec. Butyric acid and isobutyric acid was negatively correlated with low-density lipoprotein $(P<0.05)$. Interestingly, serum albumin level showed a positive correlation with acetic acid, isobutyric acid and caproic $\operatorname{acid}(P<0.05)$.

\section{Characteristics of gut microbiota in IgAN patients}

The $\alpha$-diversity, including Chaol $(P=0.0945$, Fig. 1 A), observed species $(P=0.051$, Fig. $1 \mathrm{~B})$ and Simpson $(P=$ 0.067 , Fig. $1 \mathrm{C}$ ), were approaching to the significant difference between two groups. Shannon index was different between two groups $(P=0.033$, Fig. 1D). The $\beta$ diversity was significantly different between the IgAN and normal control groups by Non-Metric MultiDimensional Scaling(NMDS) (Stress $=0.132)($ Fig. $1 \mathrm{E})$. 
Table 1 Comparison of clinical parameters between control and IgAN groups

\begin{tabular}{llll}
\hline Clinical parameters & Control group & IgAN group & $P$ value \\
\hline WBC $\left(10^{9} / \mathrm{L}\right)$ & $5.69 \pm 1.35$ & $6.83 \pm 1.78$ & 0.012 \\
Neutrophils $\left(10^{9} / \mathrm{L}\right)$ & $3.34 \pm 1.24$ & $4.26 \pm 1.36$ & 0.014 \\
Lymphocytes $\left(10^{9} / \mathrm{L}\right)$ & $1.84 \pm 0.44$ & $1.93 \pm 0.71$ & 0.549 \\
Hemoglobin(g/L) & $140.34 \pm 16.64$ & $133.93 \pm 17.83$ & 0.137 \\
Platelets $\left(10^{9} / \mathrm{L}\right)$ & $230.00(192.00,283.50)$ & $230.00(201.50,259.00)$ & 0.938 \\
Creatinine $(\mu \mathrm{mol} / \mathrm{L})$ & $61.43 \pm 13.10$ & $80.29 \pm 28.55$ & 0.003 \\
Urea nitrogen $(\mathrm{mol} / \mathrm{L})$ & $4.63 \pm 1.04$ & $5.81 \pm 1.82$ & 0.003 \\
Uric acid $(\mu \mathrm{mol} / \mathrm{L})$ & $297.57 \pm 64.69$ & $370.72 \pm 86.03$ & 0.001 \\
Albumin $(\mathrm{g} / \mathrm{L})$ & $45.35 \pm 2.52$ & $40.91 \pm 4.59$ & $<0.001$ \\
Total cholesterol/(mmol/L) & $4.35 \pm 0.58$ & $4.93 \pm 1.05$ & 0.009 \\
Triglycerides $(\mathrm{mmol} / \mathrm{L})$ & $1.01(0.77,1.33)$ & $1.31(1.01,2.18)$ & 0.007 \\
Low-density lipoprotein $(\mathrm{mmol} / \mathrm{L})$ & $2.51 \pm 0.43$ & $2.90 \pm 0.77$ & 0.025 \\
Glucose(mmol/L) & $4.71 \pm 0.43$ & $4.77 \pm 0.62$ & 0.580 \\
eGFR(mL/min·1.73 m $\left.{ }^{2}\right)$ & $103.89(99.40,114.34)$ & $101.95(72.70,123.44)$ & 0.380 \\
24-h urine protein $(\mathrm{mg} / 24 \mathrm{~h})$ & -- & $774.75(366.18,2030.60)$ & - \\
\hline
\end{tabular}

Overall, 18 bacterial phyla were recovered across the samples. The main phyla were Firmicutes (53.40\%), Bacteroidetes (36.12\%), Proteobacteria (6.89\%), Actinobacteria (3.28\%) in IgAN group, the same as those of Firmicutes (51.73\%), Bacteroidetes (43.55\%), Proteobacteria (3.08\%), Actinobacteria $(0.67 \%)$ in control group. The relative abundance of Actinobacteria in IgAN was higher than that in controls with significantly statistical difference $(P=$ 0.013).

The LEfSe analysis (Supplementary Figure S1) was used to identify the metagenomic biomarker by way of class comparison. The results of LEfSe showed that 179 bacteria taxa had biologically consistent and statistically significant differences between two groups. The 91 bacteria taxa were more abundant in normal control group, and 88 bacteria taxa were more abundant in IgA nephropathy group.

\section{Correlations between SCFAs and microbial indexes}

The correlations of SCFAs and microbial indexes detected in the IgAN patients were analyzed (Fig. 2 A). Acetic acid was positively associated with $c_{-}$Clostridia $(r=$ $0.357, P=0.008)$, o_Clostridiales $(r=0.357, P=0.008)$ and g_Eubacterium_coprostanoligenes_group $(r=0.283, \quad P=$ 0.036). Butyric acid was positively associated with $g$ Alistipes $(r=0.278, P=0.040)$. Isobutyric acid was positively associated with g_Lachnospiraceae NK4A136_group and $f \_R u m i n o c o c c a c e a e(P<0.05)$. Valeric acid was positively associated with g_Lactobacillus $(r=0.300, P=0.026)$. c_Alphaproteobacteria, o_Rhizobiales, f_Rhizobiaceae, $f$ Enterococcaceae, $g$ Intestinibacter, g_Enterococcus, g_Megamonas and $g$ _Ruminococcaceae_UCG-002 were negatively correlated with SCFAs $(P<0.05)$ (Fig. 2 A).

The LDA (Figure F) was used to identify the metagenomic biomarker by way of class comparison. Compared

Table 2 Comparison of SCFAs between control and IgAN groups

\begin{tabular}{lllr}
\hline SCFAs & Control group & IgAN group & $P$ value \\
\hline Acetic acid $(\mathrm{mmol} / \mathrm{g})$ & $79.56(66.31,135.83)$ & $36.38(30.10,65.92)$ & $<0.001$ \\
Propionic acid(mmol/g) & $6.98(3.37,8.13)$ & $4.16(3.05,5.47)$ & 0.013 \\
Butyric acid(mmol/g) & $1.45(1.12,2.14)$ & $0.66(0.49,0.96)$ & $<0.001$ \\
Isobutyric acid(mmol/g) & $7.17 \pm 5.26$ & $0.68 \pm 0.16$ & $<0.001$ \\
Valeric acid(mmol/g) & $0.28(0.16,0.36)$ & $0.19(0.09,0.37)$ & 0.129 \\
Isovaleric acid $(\mathrm{mmol} / \mathrm{g})$ & $0.53(0.39,0.76)$ & $0.39(0.28,0.67)$ & 0.095 \\
Caproic acid(mmol/g) & $0.95 \pm 0.45$ & $0.54 \pm 0.12$ & $<0.001$ \\
\hline
\end{tabular}


Table 3 Correlations between fecal SCFAs and clinical parameters

\begin{tabular}{lllc}
\hline Variable 1 & Variable 2 & r & P value \\
\hline Acetic acid & Albumin & 0.337 & 0.010 \\
Propionic acid & Total cholesterol & -0.264 & 0.045 \\
Butyric acid & Urea nitrogen & -0.316 & 0.016 \\
& Low-density lipoprotein & -0.390 & 0.002 \\
& Total cholesterol & -0.386 & 0.003 \\
& Uric acid & -0.336 & 0.010 \\
& Triglyceride & -0.390 & 0.002 \\
Isobutyric acid & Albumin & 0.410 & 0.001 \\
& Urea nitrogen & -0.299 & 0.022 \\
& Total cholesterol & -0.306 & 0.020 \\
& Triglyceride & -0.320 & 0.014 \\
& Low-density lipoprotein & -0.279 & 0.034 \\
Valeric acid & Uric acid & -0.298 & 0.022 \\
Caproic acid & Triglyceride & -0.277 & 0.036 \\
& Albumin & 0.430 & 0.001 \\
& 24-h urine protein & -0.415 & 0.025 \\
\hline
\end{tabular}

with control group, the relative abundance of $c_{-}$Clostridia, o_Clostridiales, g_Lachnospiraceae_NK4A136_ group, g_Ruminococcaceae_UCG_010, g_Alistipes and $g$ Eubacterium_coprostanoligenes_group were significantly decreased in the IgAN group. Meanwhile, the relative abundance of $c_{-}$Alphaproteobacteria, o_Rhizobiales, $f_{-}$ Rhizobiaceae, $f$ Enterococcaceae, $g$ Intestinibacter, $g$ Enterococcus, g_Megamonas and g_Ruminococcaceae UCG-002 were increased significantly in the IgAN group(Fig. 2B) .

\section{Discussion}

This study is the first to examine fecal SCFAs in patients with IgAN, and we found significant differences in SCFAs between the IgAN group and normal controls, with significantly lower levels of acetic acid, propionic acid, butyric acid, isobutyric acid and caproic acid in IgAN. There were few studies consistent with our study, where propionic acid and butyric acid levels were significantly reduced in mice with diabetic nephropathy [18]. Wang et al. [19] found significantly lower fecal SCFAs in CKD 5 than in patients with CKD stages 1-4, and negatively correlated with the renal function. Wu et al. [20] found significantly lower serum SCFAs levels in patients with CKD stages 4-5 compared to the normal group.

In our study we detected the fecal microbiota, it is a proxy of the composition of the gut microbiota [21]. There was the dysbiosis of gut microbiota in IgAN patients compared to controls, exemplified by the results of $\alpha$-diversity, $\beta$-diversity and LEfSe analysis in our study. De Angelis et al. [22] reported that the lower microbial diversity was found in IgAN patients estimated by rarefaction, Chao 1and Shannon diversity index. In agreement with our study, $\mathrm{Hu} \mathrm{X}$ et al. [23] also demonstrated that the community richness of fecal microbiota in the IgAN patients was significantly lower than that in the healthy controls.

The reason for the significant decrease of fecal SCFA in IgAN patients, was related to the decreased relative abundances of SCFA-producing bacteria compared to the normal group. The bacteria taxa of $c_{-}$Clostridia, o_Clostridiales, and g_Eubacterium_coprostanoligenes_ group were positively correlated with acetic acid, and g_Alistipes was positively correlated with butyric acid. Isobutyric acid was positively associated with $g_{-}$Lachnospiraceae NK4A136 group and $f \_$Ruminococcaceae. Furthermore, The relative abundance of those were significantly decreased in IgAN group compared to control group. Especially, Clostridia, Alistipes and Ruminococcaceae were comfirmed as important SCFA-producing bacteria [24, 25]. Clostridium was reported to produce acetate by the Wood-Ljungdahl pathway [26]. Lachnospiraceae was demonstrated to use lactate and acetate to produce butyrate [27]. Baxter NT et al. [28] found that gut microbiota with an increase in Ruminococcus or Clostridium were more likely to yield higher butyrate concentrations.

The relative abundance of $c_{-}$Alphaproteobacteria, $o$ Rhizobiales, f_Rhizobiaceae, f_Enterococcaceae, $g$ Intestinibacter, $g$ Enterococcus, $g$ _Megamonas and $g$ Ruminococcaceae_UCG-002 were negatively correlated with SCFAs. SCFAs often exhibited broad-spectrum antimicrobial activity, mainly due to its ability to penetrate and destroy microorganisms. It has been demonstrated that SCFA has both bactericidal and antibacterial functions against oral microorganisms. In vitro experiments have demonstrated that butyric acid can inhibit Salmonella infection [29].SCFAs are relatively inert to the bacteria that produce them, but can effectively inhibit the growth of other bacteria [30]. In addition to its direct effect on the intestinal barrier, SCFA can lower the $\mathrm{pH}$ in the intestinal lumen, which can directly promote the growth of commensal bacteria and inhibit the proliferation of pathogenic bacteria [31].

In this study, the negative correlations between SCFAs and kidney injury-related indicators were found. Butyric acid was negatively correlated with urea acid and urea nitrogen; caproic acid showed negative correlation with 24-h urine protein level. Similar to the findings of previous studies, Wang et al. [19] reported that the serum acetate and butyrate levels in CKD 5 were significantly lower than those in CKD 1-4 patients. Wu et al. [20] found that compared with the normal group, the serum propionic acid level of CKD4-5 patients was significantly lower, and it can be used to distinguish patients with severe renal impairment from the normal group. In the 
A

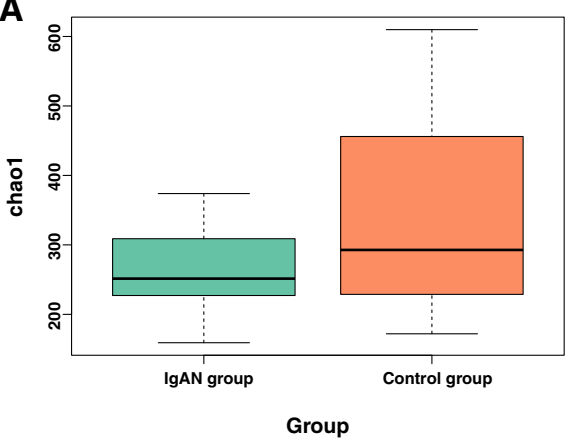

C

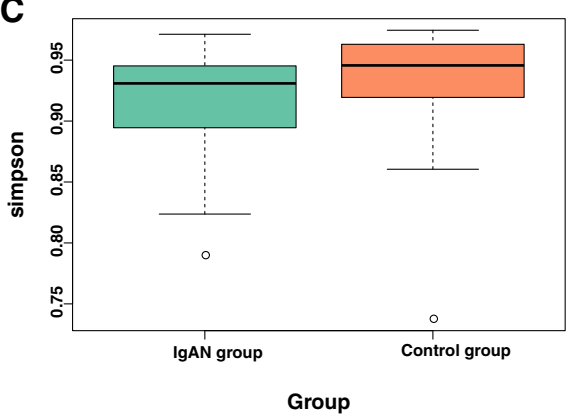

B

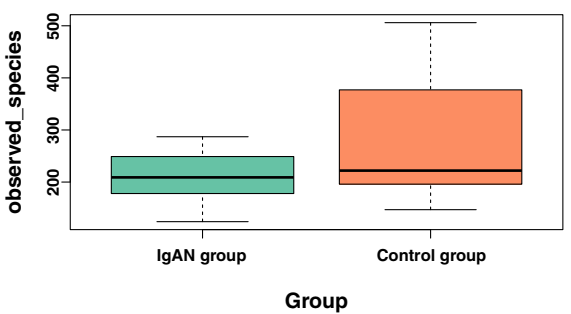

D

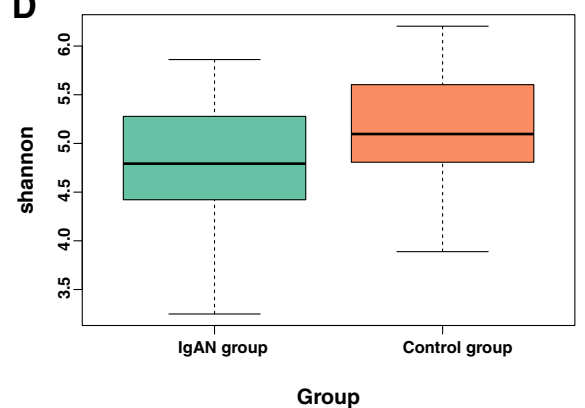

E

NMDS Plot

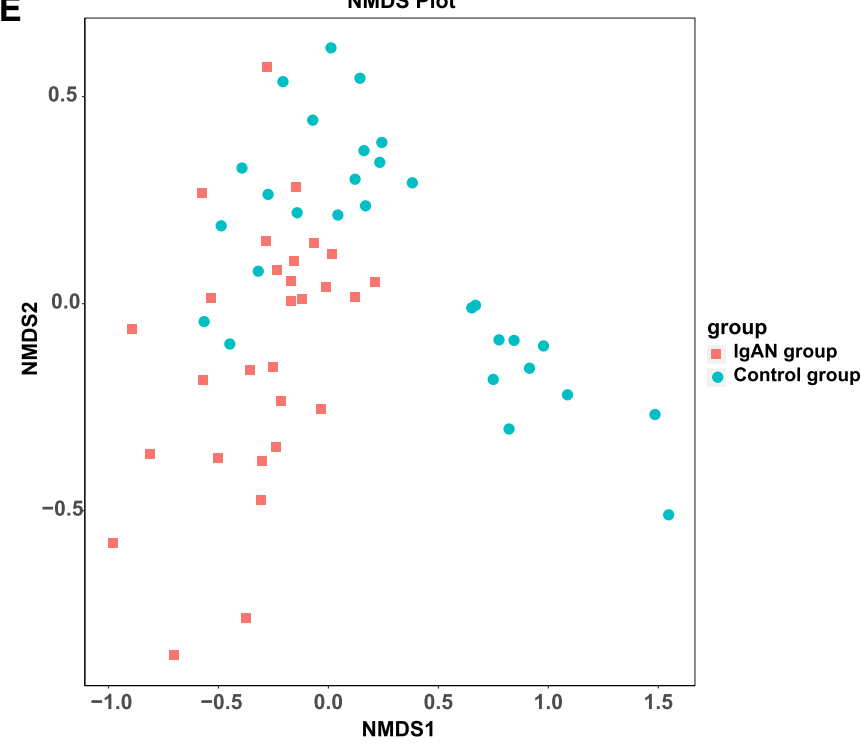

Fig. 1 The gut microbiota composition of IgAN patients was significantly different from that of the control. The a-diversity of the microbiota presented as Chao1 $(P=0.0945, \mathbf{A})$, observed species $(P=0.051$, B), Simpson $(P=0.067, \mathbf{C})$ and the Shannon index $(P=0.033, \mathbf{D})$. The $\beta$-diversity in the IgAN and control groups was calculated by NMDS (Stress $=0.132)($ Fig. 1E)

study conducted by Zhang $\mathrm{L}$ et al., SCFAs were increased and blood urea nitrogen level was decreased following resistant starch intake [32]. SCFAs exerted its effects on the "entero-renal axis" mainly through G protein-coupled receptors (GPR) and direct inhibition of Histone deacetylase (HDAC) [33]. SCFAs also exerted anti-bacterial, anti-inflammatory and anti-oxidative effects in numerous studies [34].
The role of short-chain fatty acids in IgAN remained unclear. Previous studies have confirmed that SCFAs modulated inflammation both in intestinal and extraintestinal environments and possessed multifarious effects against inflammatory bowel disease and allergic airway disease by decreasing inflammatory response. [35, 36]. Meanwhile, a strong relationship between IgAN and intestinal inflammation was reported, such as 

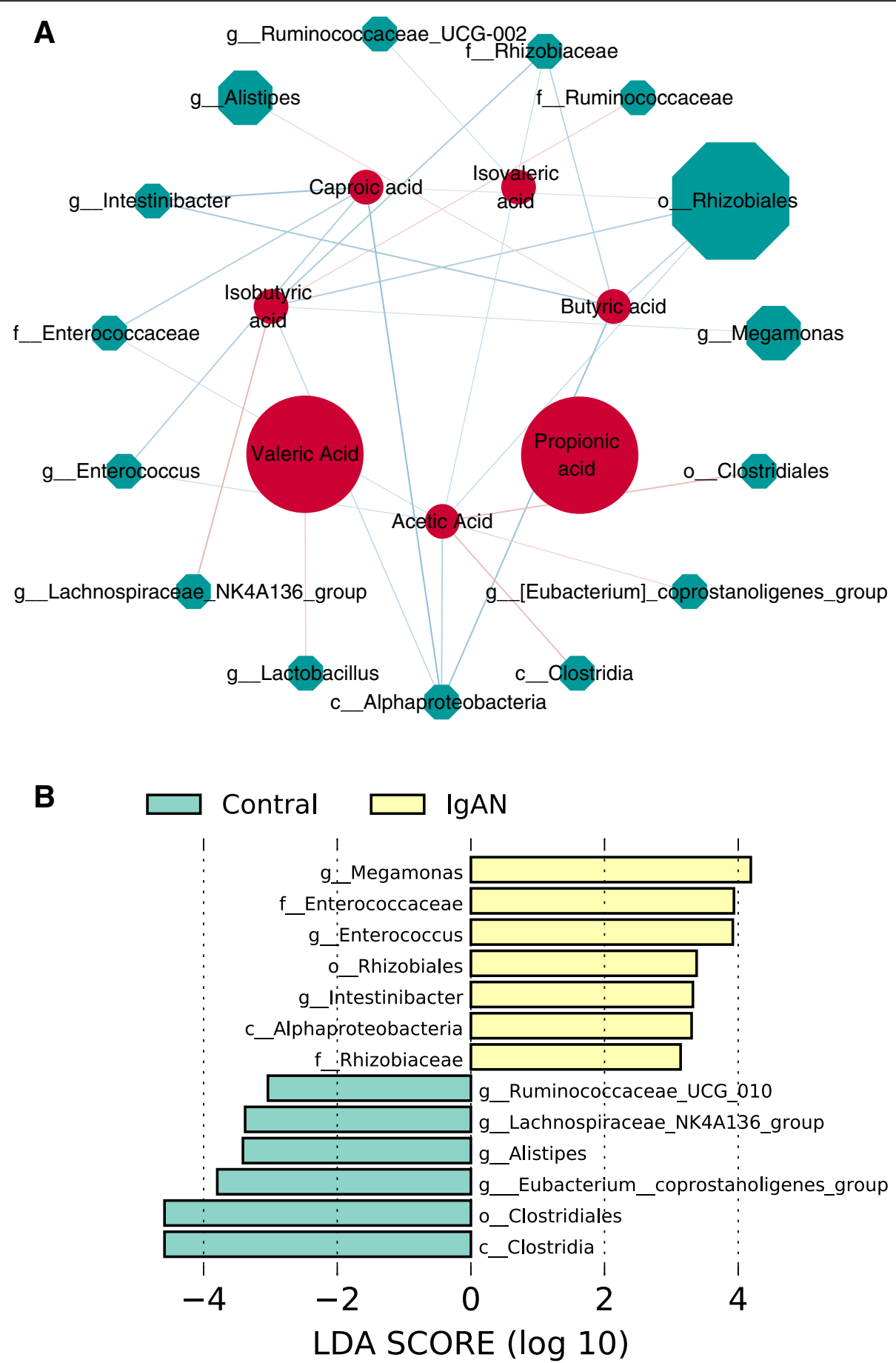

Fig. 2 Correlations between SCFAs and microbial indexes. The network diagram of the correlation analysis of microbial indexes and SCFAs (A), red represents SCFAs, and green microbial indexes. The color of the edge shows the correlation coefficient (red positive and blue negative)( $P<$ 0.05). The node size represents the centrality, that is, the number of consecutive starting from it. LDA was performed to determine the difference of the SCFAs related microbial taxa in two groups (B). Significantly different bacteria with LDA scores $\geq 2.0$ were diagrammed on cladogram

inflammatory bowel diseases [37] and coeliac disease [38]. Qin D et al., found that P-aIgA1 (aggregated IgA1 from IgAN patients) promoted the proliferation of human renal mesangial cells (HMCs), and markedly increased the protein levels of HDAC1 in the cells. Pretreatment with SCFAs inhibitor of HDAC inpartially reversed P-aIgA1-induced cell proliferation and extracellular matrix synthesis in HMCs [39]. 
Meanwhile, a strong inverse relationship between SCFAs and blood lipids. Propionic acid, butyric acid and isobutyric acid showed negative correlations with total cholesterol $(P<0.05)$. Butyric acid, isobutyric acid and valeric acid showed negative correlation with triglyceridec. Butyric acid and isobutyric acid was negatively correlated with low-density lipoprotein $(P<0.05)$. Previous studies have found that SCFAs also have a role in regulating blood lipids. Propionic acid and butyric acid inhibited isoprenaline- and adenosine deaminasestimulated lipolysis in the presence of phosphodiesterase (PDE3) inhibitors as well as propionic acid and butyric acid inhibited basal and insulin-stimulated de novo lipogenesis, which was associated with increased phosphorylation, thereby inhibiting the activity of HMG-CoA, the rate-limiting enzyme for fatty acid synthesis. Moreover, SCFAs have an effect on fat storage and mobilization in rat primary adipocytes, thus possibly contributing to healthier adipocyte contracts and improving energy metabolism and reducing circulating free fatty acids [40].

Interestingly, serum albumin level showed a positive correlation with acetic acid, isobutyric acid and caproic acid significantly. Although we didn't found the report on SCFAs and albumin directly related, there were a few studies on SCFAs and malnutrition [41, 42]. Studies in mouse models revealed that gut-derived SCFAs were actively metabolized and that propionate, butyrate, and acetate played an important role as substrates for glucose metabolism [43]. Recent study [44] presented the potential role of the gut microbiota in anorexia nervosa(AN) by evaluating fecal microbiota composition and SCFAs. Compared to normal-weight participants, the concentrations of butyrate-producing Roseburia spp were reduced in patient with AN.

There are several limitations in this study. First, we found that gut microbiota and SCFAs were involved in IgAN, but the possible mechanisms and pathways have not been elucidated. Second, the sample size was relative small, and the multiple confounders were not controlled for all correlation analyses. Our data should be interpreted with caution, and further studies are needed to verify the role of SCFA produced by the intestinal flora in IgAN.

\section{Conclusions}

The results revealed that fecal SCFAs levels were significantly lower in patients with IgAN, which was related to dysbiosis in IgAN patients. There was a significant correlation between clinical parameters (urea nitrogen, uric acid, proteinuria and lipids ) and SCFAs, which also provides a new basis for future exploration of the role of the entero-renal axis in IgAN.

\section{Supplementary Information}

The online version contains supplementary material available at https://doi. org/10.1186/s12882-021-02414-x.

\section{Additional file 1:}

Additional file 2:Supplementary Figure S1. The Cladogram based on LEfSe results of the IgAN and control groups. The yellow points represent the increased taxa in control group, while the blue points represent the increased taxa in IgAN group.

Additional file 2:Supplementary Figure S1. The Cladogram based on LEfSe results of the IgAN and control groups. The yellow points represent the increased taxa in control group, while the blue points represent the increased taxa in IgAN group.

\section{Acknowledgements}

Not applicable.

\section{Authors' contributions}

$\mathrm{L}-\mathrm{XC}$ and $\mathrm{B}-\mathrm{BX}$ conceived and designed the research; L-XC and K-DC searched the literature; L-XC and K-YW extracted data and results were verified by K-DC and QL; L-XC, K-DC and K-YW analyzed data and prepared tables and figures; L-XC and K-DC drafted the manuscript; B-BX, K-DC and QL critically reviewed and revised the manuscript and contributed to the discussion; and all authors read and approved the final version of the submitted manuscript.

\section{Funding}

General Project of Natural Science Foundation of Zhejiang Province (No.: LY20H050005); Ningbo Science and Technology Plan Project (No:

2019A610264); Medical Scientific Research Foundation of Zhejiang Province, China (No.: 2019KY174) and Ningbo Public Service Techology Foundation, China (No.: 2019C50084).

Availability of data and materials

The data are available from the corresponding author on reasonable request.

\section{Declarations}

Ethics approval and consent to participate

The study was conducted according to the Declaration of Helsinki. It was approved by the Medical Ethics Committee of Ningbo Hwamei Hospital (No.PJ-NBEY-KY-2018-046-01). All participants provided written informed consent in this study.

Consent for publication

Not applicable.

\section{Competing interests}

The authors declare that they have no competing interests.

Received: 29 March 2021 Accepted: 17 May 2021

Published online: 03 June 2021

References

1. Yang Y, Tang X, Yang Y, Li X, Li L, Huang K, et al. Glomerular C4 deposition and glomerulosclerosis predict worse renal outcomes in Chinese patients with IgA nephropathy. Ren Fail. 2020;42(1):629-637.

2. Tao J, Mariani L, Eddy S, Maecker $\mathrm{H}$, Kambham N, Mehta K, et al. JAK-STAT Activity in Peripheral Blood Cells and Kidney Tissue in IgA Nephropathy. Clinical Journal of the American Society of Nephrology. 2020; 15(7): 973982.

3. Cheng W, Zhou X, Zhu L, Shi S, LV J, Liu L, et al. Polymorphisms in the nonmuscle myosin heavy chain 9 gene (MYH9) are associated with the progression of IgA nephropathy in Chinese. Nephrology Dialysis Transplantation. 2011; 26(8): 2544-2549.

4. McGrogan A, Franssen CF, de Vries CS. The incidence of primary glomerulonephritis worldwide: a systematic review of the literature. Nephrol Dial Transplant. 2011; 26(2): 414-430. 
5. Moriyama T, Tanaka K, Lwasaki C, Oshima Y, Ochi A, Kataoka H, et al. Prognosis in IgA nephropathy: 30 year analysis of 1012 patients at a single center in Japan. PLoS One. 2014; 9(3): e91756.

6. Coppo R. The Gut-Renal Connection in IgA Nephropathy. Semin Nephrol. 2018; 38(5):504-512.

7. Yang T, Richards EM, Pepine CJ, Raizada MK. The gut microbiota and the brain-gut-kidney axis in hypertension and chronic kidney disease. Nature Reviews Nephrology. 2018; 14(7):442-456.

8. Coppo R. The gut-kidney axis in IgA nephropathy: role of microbiota and diet on genetic predisposition. Pediatric Nephrology. 2018; 33(1): 53-61.

9. Yu H, Li R, Huang H, Yao R, Shen S. Short-Chain Fatty Acids Enhance the Lipid Accumulation of 3T3-L1 Cells by Modulating the Expression of Enzymes of Fatty Acid Metabolism. Lipids. 2018; 53(1):77-84.

10. Hernández MAG, Canfora EE, Jocken JWE, Blaak EE. The Short-Chain Fatty Acid Acetate in Body Weight Control and Insulin Sensitivity. Nutrients. 2019; 11(8):1943

11. Felizardo RJF, Watanabe IKM, Dardi P, Rossoni LV, Câmara NOS. The interplay among gut microbiota, hypertension and kidney diseases: The role of short-chain fatty acids.Pharmacol Res. 2019;141:366-377.

12. Onal EM, Afsar B, Covic A, Vaziri ND, Kanbay M. Gut microbiota and inflammation in chronic kidney disease and their roles in the development of cardiovascular disease. Hypertens Res. 2019;42(2):123-140.

13. Huang $W$, Zhou $L$, Guo $H, X u Y, X u Y$. The role of short-chain fatty acids in kidney injury induced by gut-derived inflammatory response. Metabolism. 2017, 68:20-30.

14. Huang W, Guo HL, Deng X, Zhu TT, Xiong JF, Xu YH, et al. Short-Chain Fatty Acids Inhibit Oxidative Stress and Inflammation in Mesangial Cells Induced by High Glucose and Lipopolysaccharide. Exp Clin Endocrinol Diabetes. 2017;125(2):98-105.

15. Gu J, Huang W, Zhang W, Zhao T, Gao C, Gan W, et al. Sodium butyrate alleviates high-glucose-induced renal glomerular endothelial cells damage via inhibiting pyroptosis. Int Immunopharmacol. 2019;75:105832

16. Levey AS, Stevens LA, Schmid CH, Zhang YL, Castro 3rd AF, Feldman HI, et al. A new equation to estimate glomerular filtration rate. Ann Intern Med. 2009;150 (9):604-612

17. Segata N, Izard J, Waldron L, Gevers D, Miropolsky L, Garrett WS, et al. Metagenomic biomarker discovery and explanation. Genome Biol.2011;12: R60.

18. Li Y, Su X, Gao Y, Lv C, Gao Z, Liu Y, et al. The potential role of the gut microbiota in modulating renal function in experimental diabetic nephropathy murine models established in same environment. Biochim Biophys Acta Mol Basis Dis.2020; 1866: 165764.

19. Wang S, Lv D, Jiang S, Jiang J, Liang M, Hou F, et al. Quantitative reduction in short-chain fatty acids, especially butyrate, contributes to the progression of chronic kidney disease. Clin Sci(Lond) 2019; 133: 1857-1870.

20. Wu IW, Gao SS, Chou HC, Yang HY, Chang LC, Kuo YL et al. Integrative metagenomic and metabolomic analyses reveal severity-specific signatures of gut microbiota in chronic kidney disease. Theranostics. 2020; 10: 5398-5411.

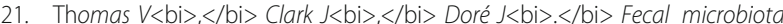
analysis: an overview of sample collection methods and sequencing strategies. Future Microbiol<bi $>$.</bi $>2015<$ bi $>;</$ bi $>10<$ bi $>(</$ bi $>9<b i>):</ b i>1485-$ $504<\mathrm{bi}>$. $</$ bi $>$

22. De Angelis M, Montemurno E, Piccolo M, Vannini L, Lauriero G, Maranzano $\checkmark$, et al. Microbiota and metabolome associated with immunoglobulin A nephropathy (IgAN). PLoS One. 2014; 9(6):e99006.

23. Hu X, Du J, Xie Y, Huang Q, Xiao Y, Chen J, et al. Fecal microbiota characteristics of Chinese patients with primary lgA nephropathy: a crosssectional study. BMC Nephrol. 2020;21(1):97.

24. Tammali R, Seenayya G, Reddy G. Fermentation of cellulose to acetic acid by Clostridium lentocellum SG6: induction of sporulation and effect o buffering agent on acetic acid production.Lett Appl Microbiol. 2003;37(4): 304-8.

25. Guilin Z, Pengyu Z, Wei L, Fengqi $H$, Chen F, Yu Y, et al. Reduction of gut microbial diversity and short chain fatty acids in BALB/C mice exposure to microcystin-LR. Ecotoxicology. 2020; 29(9):1347-1357.

26. Koh A, De Vadder F, Kovatcheva-Datchary P, Bäckhed F. From Dietary Fiber to Host Physiology: Short-Chain Fatty Acids as Key Bacterial Metabolites. Cell. 2016;165(6):1332-1345.

27. Louis P, Flint HJ. Diversity, metabolism and microbial ecology of butyrateproducing bacteria from the human large intestine. FEMS Microbiol Lett. 2009; 294(1):1-8.
28. Baxter NT, Schmidt AW, Venkataraman A, Kim KS, Waldron C, Schmidt TM. Dynamics of Human Gut Microbiota and Short-Chain Fatty Acids in Response to Dietary Interventions with Three Fermentable Fibers. mBio. 2019; 10(1): e02566-18.

29. Fernández-Rubio C, Ordóñez C, Abad-González J, Garcia-Gallego A, Honrubia MP, Mallo JJ, et al. Butyric acid-based feed additives help protect broiler chickens from Salmonella Enteritidis infection. Poult Sci. 2009;88(5): 943-8.

30. Alva-Murillo N, Ochoa-Zarzosa A, López-Meza JE. Short chain fatty acids (propionic and hexanoic) decrease Staphylococcus aureus internalization into bovine mammary epithelial cells and modulate antimicrobial peptide expression.Vet Microbiol. 2012;155(2-4):324 - 31

31. Greenberg NA, Gassull MA, Meier R.Short-chain fatty acids: ready for prime time?Nutr Clin Pract.2006;21(4):351 - 66

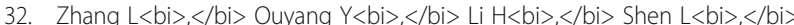
$\mathrm{Ni} Y<b i>,</$ bi $>$ Fang $\mathrm{Q}<\mathrm{bi}>$, et $\mathrm{al} .</ \mathrm{bi}>$ Metabolic phenotypes and the gut microbiota in response to dietary resistant starch type 2 in normal-weight subjects: a randomized crossover trial. Sci Rep $<$ bi $>$. $</$ bi $>2019<$ bi $>;</$ bi $>9<$ bi $>(</$ bi $>1<$ bi $>):</$ bi $>4736<$ bi $>$. $</$ bi $>$

33. Marzocco S, Fazeli G, Di Micco L, Autore G, Adesso S, Dal Piaz F, et al. Supplementation of short-chain fatty acid, sodium propionate, in patients on maintenance hemodialysis: beneficial effects on inflammatory parameters and gut-derived uremic toxins, a pilot study (PLAN study). J Clin Med. 2018;7.

34. Lin MY, de Zoete MR, van Putten JP, Strijbis K. Redirection of epithelial immune responses by short-chain fatty acids through inhibition of histone deacetylases. Front Immunol. 2015;6:554.

35. Aurélien T, Gollwitzer ES, Koshika Y, Sichelstiel AK, Sprenger N, Ngom-Bru C, et al. Gut microbiota metabolism of dietary fiber influences allergic airway disease and hematopoiesis. Nat Med.2014;20(2):159 - 66

36. Galvez J, Rodrãguez-Cabezas ME, Zarzuelo A. Effects of dietary fiber on inflammatory bowel disease. Mol Nutr Food Res.2005;49(6):601-8.

37. Ambruzs JM, Walker PD, Larsen CP. The histopathologic spectrum of kidney biopsies in patients with inflammatory bowel disease. Clin J Am Soc Nephrol.2014; 9: 265-270.

38. Welander A, Sundelin B, Fored M, Ludvigsson JF. Increased risk of IgA nephropathy among individuals with celiac disease. J Clin Gastroenterol. 2013; 47: 678-683.

39. Dai Q, Liu J, Du Y, Hao X, Ying J, Tan Y, et al. Histone deacetylase inhibitors attenuate $P$-algA1-induced cell proliferation and extracellular matrix synthesis in human renal mesangial cells in vitro. Acta Pharmacol Sin. 2016; $37(2): 228-34$.

40. Heimann E, Nyman M, Degerman E. Propionic acid and butyric acid inhibit lipolysis and de novo lipogenesis and increase insulin-stimulated glucose uptake in primary rat adipocytes. Adipocyte.2014;4(2):81-88.

41. de Clercq $\mathrm{NC}<\mathrm{bi}>,</ \mathrm{bi}>$ Groen $\mathrm{AK}<\mathrm{bi}>,</ \mathrm{bi}>$ Romijn $\mathrm{JA}<\mathrm{bi}\rangle,</ \mathrm{bi}>$ Nieuwdorp $\mathrm{M}<\mathrm{bi}>.</ \mathrm{bi}>$ Gut Microbiota in Obesity and Undernutrition. Adv Nutr. 2016 Nov $15<\mathrm{bi}>:</$ bi $>7<$ bi $>(</$ bi $>6<$ bi $>):</$ bi $>1080-1089<b i>$. $</$ bi $>$

42. Pekmez $C \mathrm{~T}<\mathrm{bi}>$, $</$ bi $>$ Dragsted $\mathrm{LO}<\mathrm{bi}>$, $</ \mathrm{bi}>$ Brahe $\mathrm{LK}<\mathrm{bi}>,</ \mathrm{bi}>$ Gut microbiota alterations and dietary modulation in childhood malnutrition The role of short chain fatty acids. Clin Nutr $<$ bi $><</$ bi $>2019<$ bi $>$ Apr; $</$ bi $>38<$ bi $>(</$ bi $>2<$ bi $>):</$ bi $>615-630<$ bi $>$. $</$ bi $>$

43. den Besten $G$, Lange $K$, Havinga R, van Dijk TH, Gerding A, van Eunen $K$, et al. Gut-derived short-chain fatty acids are vividly assimilated into host carbohydrates and lipids. Am J Physiol Gastrointest Liver Physiol. 2013; 305: G900-10.

44. Mack I, Cuntz U, Grämer C, Niedermaier S, Pohl C, Schwiertz A, et al. Weight gain in anorexia nervosa does not ameliorate the faecal microbiota, branched chain fatty acid profiles, and gastrointestinal complaints. Sci Rep. 2016:6:26752.

\section{Publisher's Note}

Springer Nature remains neutral with regard to jurisdictional claims in published maps and institutional affiliations. 